\title{
Lung Ultrasound in Ventilator Associated Pneumonia: Light or More Shadow at Diagnosis?
}

\author{
Abdo-Cuza A ${ }^{1 *}$, Díaz-Águila $\mathrm{H}^{2}$, Valdés-Suarez $\mathrm{O}^{3}$, Castellanos-Gutiérrez $\mathrm{R}^{1}$, Suárez-López $\mathrm{J}^{4}$ and \\ Machado-Martínez $\mathbf{R}^{1}$ \\ ${ }^{1}$ Intensive Care Unit, Medical Surgical Research Center, Havana, Cuba \\ ${ }^{2}$ University Hospital of Sagua la Grande, Villa Clara, Cuba \\ ${ }^{3}$ University General Hospital Iván Portuondo, Artemis, Cuba \\ ${ }^{4}$ Hermanos Ameijeiras Hospital, Havana Cuba
}

${ }^{*}$ Corresponding author: Abdo-Cuza Anselmo, Intensive Care Unit, Medical Surgical Research Center, Havana, Cuba, Tel: +5378581511 ; E-mail: aaabdo@infomed.sld.cu

Received date: November 28, 2018; Accepted date: January 18, 2019; Published date: January 22, 2019

Citation: Abdo-Cuza A, Díaz-Águila H, Valdés-Suarez O, Castellanos-Gutiérrez R, Suárez-López J, et al. (2019) Lung Ultrasound in Ventilator Associated Pneumonia: Light or More Shadow at Diagnosis?. Arch Med Vol No:11 Iss No:1:1

Copyright: (02019 Abdo-Cuza A, et al. This is an open-access article distributed under the terms of the Creative Commons Attribution License, which permits unrestricted use, distribution, and reproduction in any medium, provided the original author and source are credited.

\section{Abstract}

Ventilator-associated pneumonia (VAP) is the most common health care-associated infection in intensive care units (ICU). Its appearance causes an increase in stay, mortality and economic costs. The traditional diagnostic criteria (clinical, radiological and microbiological) are a matter of growing controversy (the poor reliability of radiological criteria in ICU patients has been recognized). For a few years, lung ultrasound has occupied a vital place in the diagnostic arsenal of intensive medicine. Review articles and Meta-analyzes on lung ultrasound in the diagnosis of pneumonia have confirmed the usefulness of the method. The main ultrasonographic characteristics of VAP are the presence of pulmonary condensation and air bronchogram, with a sensitivity of $100 \%$ and a specificity of $80 \%$. The image of condensation may correspond to other diagnoses such as pulmonary atelectasis caused by mechanical obstruction or compression, tumor consolidation and pulmonary embolism. On the other hand there are technical limitations (obese patients, patients with drainages and the location of the lesion) that compromise the diagnostic certainty. In conclusion, lung ultrasound is useful but has not yet provided all the light necessary for the successful diagnosis of VAP in ICUs.

Keywords: Ultrasonography; Ventilator associated pneumonia; Health care associated infections; Critical care

\section{Introduction}

Ventilator-associated pneumonia (VAP) is the most common health care-associated infection in intensive care units (ICU).
Its appearance causes an increase in stay, mortality and economic costs [1].

The traditional diagnostic criteria are clinical, radiological and microbiological [2]. Around them there have been growing controversies; thus, for example, the poor reliability of radiological criteria has been recognized in ICU patients where there may be other situations (such as atelectasis or pulmonary edema), other than an inflammatory condensation and that share radiological signs. For this reason the Center for Disease Prevention and Control (CDC) of the United States, implemented in 2013 a system for monitoring events associated with ventilation, where the VAP was one of them [3]. In order to decrease the subjectivity and variability of the traditional radiological criteria, the chest radiograph was rejected as a diagnostic criterion. The major criteria are related to objective parameters of the type of end-expiration positive pressure values and fraction of oxygen in the inspired air. An interesting study by Ego et al. [4] demonstrated the variability in the diagnosis of VAP according to the criteria used. The authors report incidence rates from $4 \%$ to $42 \%$ according to the use of six of the most popular definitions, and variations from $0 \%$ to $44 \%$ modeling up to 89 combinations of criteria. At present there is no universalization of the criteria of the $C D C$ and the absence of reliable and universal diagnostic criteria, continues as an unresolved problem.

Computed tomography although it is cited as gold standard radiological criteria, is a diagnostic method that requires the transfer of the patient usually at risk, in addition to the cost and exposure to ionizing radiation; therefore, in the opinion of the authors, it should not be the study of choice for radiological diagnosis of VAP in the ICU.

Since a few years ago, a method discarded at the beginning for the imaging study of the lung and its covers, has been gaining a vital place in the diagnostic arsenal of intensive medicine: pulmonary ultrasound [5-7]. Alzahrani et al. [8] 
published a systematic review and meta-analysis on the use of pulmonary ultrasound in the diagnosis of pneumonia and concluded that it was an accurate tool, easily available, low cost and free of radiological risk.

\section{Materials and Methods}

In non-pathological pulmonary ultrasound, the pulmonary glide and $A$ lines are visualized. The first corresponds to the sliding of the visceral pleura against the parietal pleura and the A lines are repetitive artifacts of horizontal reverberation parallel to the pleural line and generated by the sub pleural air normally present in the alveoli (Figure 1A).

The $B$ lines are comet-like, hyper echoic artifacts that arise from the pleural line and propagate vertically, erasing the $A$ lines and moving when the pulmonary slip is present (Figure 1B). They indicate partial loss of pulmonary aeration and represent the thickening of sub pleural septa by deposition of fibrous tissues, inflammatory cells, or pulmonary edema.

On ultrasound examination, pulmonary consolidation is visualized with a tissue-like pattern (reminiscent of the liver), with limits that can be formed from the pleural line or a pleural effusion (if present) and the aerated lung (Figure 1C). The presence of pulmonary consolidation indicates complete loss of pulmonary aeration because the alveolar space is filled with exudates and cell debris or is collapsed by the proliferation of neoplastic tissue or pleural effusion. The presence of this pattern may correspond to pneumonia, atelectasis, contusion or acute respiratory distress syndrome.

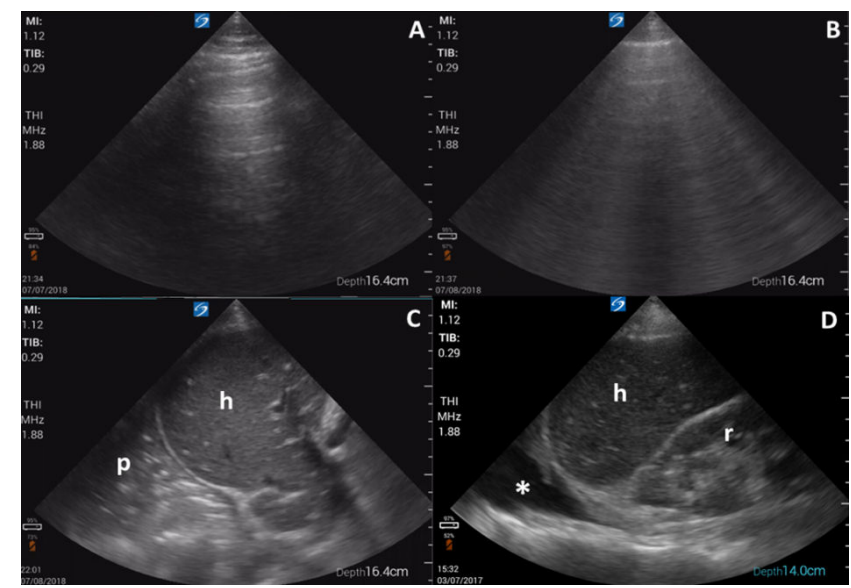

Figure 1: Lung ultrasound images obtained with 1-5 $\mathrm{MHz}$ sector transducer in upper and anterior regions ( $A$ and $B$ ), and lateral and inferior right ( $C$ and $D)$; $A$. Pattern of lines $A$; B. Pattern of lines B; C. Pattern of pulmonary condensation, $\mathrm{h}$ : liver, $\mathrm{p}$ : pulmonary parenchyma; $\mathrm{D}$. Image compatible with pleural effusion, marked with an asterisk $\left({ }^{*}\right)$, h: liver, $r$ : right kidney.

In areas of condensation, the presence of air or fluid bronchogram can be detected (Figure 2A). The ultrasound detection of a dynamic air bronchogram is useful to differentiate obstructive atelectasis from pneumonia. This phenomenon consists of an inspiratory reinforcement that is produced by the entry of air during inspiration, to the bronchial tree, (Figures 2B-2D).

The pleural effusion is identified as an anechoic area on ultrasound and may accompany infectious, inflammatory or cardiovascular conditions (Figure 1D).

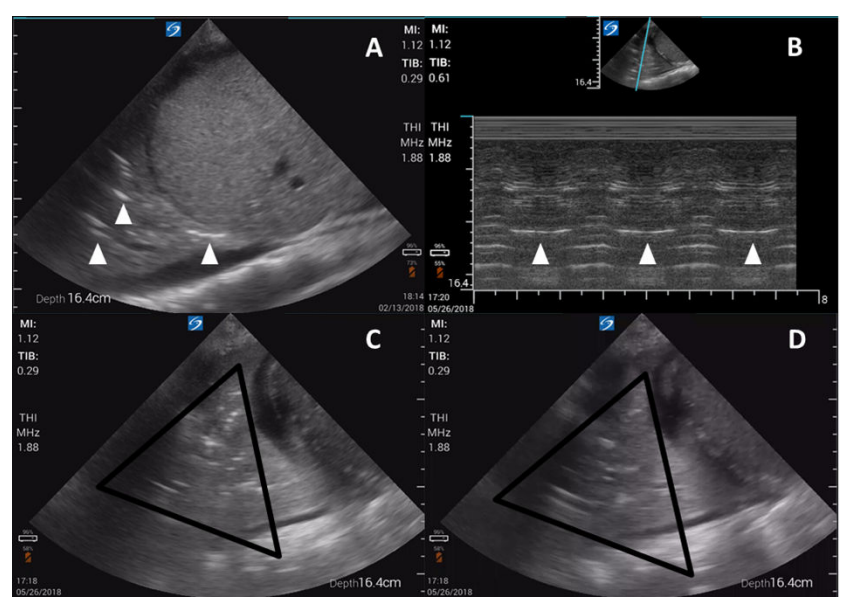

Figure 2: Lung ultrasound images obtained with 1-5 $\mathrm{MHz}$ sector transducer in the right and lateral region. A. Pattern of pulmonary condensation and air bronchograms, marked with white arrows; $\mathrm{B}$. M-mode image showing the change of the bronchogram with respiration (dynamic air bronchogram); CD. Sequences of a moving image where two moments of the respiratory cycle are visualized and the variation of bronchograms within the same space delimited by a triangle with black borders, compatible with dynamic air bronchogram.

\section{Results and Discussions}

The ultrasonographic characteristic of VAP is the presence of pulmonary condensation and air bronchogram, with a sensitivity of up to $100 \%$ and a specificity of around $60 \%$ [9]. It should be noted that the condensation image may correspond to a group of diagnoses such as pneumonia, pulmonary atelectasis caused by mechanical obstruction or compression, tumor consolidation, or pulmonary embolism. Misdiagnosis of pneumonia has been reported in $17 \%-26 \%$ of patients in the ICU [10].

It is of vital importance to know a group of limitations of the technique, among them the difficulty of image in obese patients with thick thoracic wall, patients with pleural calcifications, non-cooperative patients, and patients with bandages or thoracic drainages. It is known that around $20 \%$ of the lung surface is not visualized by ultrasound due to the interposition of the thoracic cage [11].

The efficiency of the ultrasound detection of consolidation may also be compromised by the size of the lesion and by the distance to the pulmonary surface. Small consolidations measuring less than $20 \mathrm{~mm}$, which are located posteriorly and far from the pleura cannot be detected by ultrasound. 
Additionally, there is a need for adequate professional training for the performance and interpretation of the exam.
In Table 1, the main methods used to diagnose VAP are presented as a comparison.

Table 1: Comparison between the most used methods for the diagnosis of pneumonia associated with mechanical ventilation (associated with clinical and microbiological criteria) in intensive care units.

\begin{tabular}{|l|l|l|l|l|}
\hline & Auscultation & Chest X-ray & Axial tomography & Ultrasound \\
\hline Cost & $\mathrm{x}$ & $\mathrm{xxx}$ & $\mathrm{xxxxx}$ & $\mathrm{xx}$ \\
\hline Availability & $\mathrm{xxxxx}$ & $\mathrm{xxx}$ & $\mathrm{x}$ & $\mathrm{xxxx}$ \\
\hline Transfer requirement & - & - & $\mathrm{xxxxx}$ & - \\
\hline Radiation & - & $\mathrm{xx}$ & $\mathrm{xxxxx}$ & - \\
\hline Repeatability & $\mathrm{xxxxx}$ & $\mathrm{xxx}$ & $\mathrm{x}$ & $\mathrm{xxxx}$ \\
\hline Learning & $\mathrm{xx}$ & $\mathrm{xx}$ & $\mathrm{xxxx}$ & $\mathrm{xx}$ \\
\hline Diagnostic protocols & $\mathrm{xxxxx}$ & $\mathrm{xxxxx}$ & $\mathrm{xxxxx}$ & $\mathrm{xx}$ \\
\hline Sensitivity & $6 \%-21 \%$ & $23 \%-72 \%$ & Gold standard & $60 \%-100 \%$ \\
\hline Specificity & $91 \%-96 \%$ & $27 \%-83 \%$ & Gold standard & $83 \%-90 \%$ \\
\hline
\end{tabular}

At present, some researchers have proposed pulmonary ultrasound as a substitute for chest radiography, as a radiological method for the diagnosis of VAP. Zagli et al. [12] evaluated a new diagnostic score, CEPPIS (chest echography and procalcitonin pulmonary infection score), based on the combination of procalcitonin and pulmonary ultrasound for the diagnosis of VAP. The CEPPIS sensitivity was higher $(80.5 \%)$ than CPIS (clinical pulmonary infection score) $39.8 \%$ and shared similar specificity ( 85.2 vs. $83.3 \%$ ). As a limitation, we declare that the ideas presented in this article represent only the opinions of the authors. More studies are needed to validate the usefulness of ultrasound in the diagnosis of VAP. The authors consider that endpoints such as antibiotic use, days of ventilation and survival should be evaluated in the different diagnostic methods.

\section{Conclusions}

The authors of this article recognize the utility of pulmonary ultrasound for the diagnosis of VAP. Clinical practice in the intensive care scenario appears to be the best imbalance-costfeasibility-benefit method. However, to date, the diagnostic certainty of VAP requires collective clinical trial that must be supplemented with clinical and microbiological laboratory studies. The existence of homogeneous protocols for the use of pulmonary ultrasound is necessary, which will allow for accurate analysis in the future. In conclusion pulmonary ultrasound is useful but it still does not provide all the necessary light for the successful diagnosis of VAP in the ICU.

\section{References}

1. Sousa AS, Ferrito C, Paiva JA (2018) Intubation-associated pneumonia: An integrative review. Intensive Crit Care Nurs 44: 45-52.

2. Fan Y, Gao F, Wu Y, Zhang J, Zhu M, et al. (2016) Does ventilatorassociated event surveillance detect ventilator-associated pneumonia in intensive care units? A systematic review and meta-analysis. Crit Care 20: 338.

3. Cocoros NM, Klompas M (2016) Ventilator-associated events and their prevention. Infect Dis Clin North Am 30: 887-908.

4. Ego A, Preiser JC, Vincent JL (2015) Impact of diagnostic criteria on the incidence of ventilator-associated pneumonia. Chest 147: 347-355.

5. Lichtenstein DA, Malbrain MLNG (2017) Lung ultrasound in the critically ill (LUCI): A translational discipline. Anaesthesiol Intensive Ther 49: 430-436.

6. Lichtenstein D (2017) Novel approaches to ultrasonography of the lung and pleural space: where are we now? Breathe Sheff 13: 100-111.

7. Staub LJ, Biscaro RRM, Maurici R (2018) Accuracy and applications of lung ultrasound to diagnose ventilatorassociated pneumonia: A systematic review. J Intensive Care Med 33: 447-455.

8. Alzahrani SA, Al-Salamah MA, Al-Madani WH, Elbarbary MA (2017) Systematic review and meta-analysis for the use of ultrasound versus radiology in diagnosing of pneumonia. Crit Ultrasound J 9: 6.

9. Berlet T, Etter R, Fehr T, Berger D, Sendi P, et al. (2015) Sonographic patterns of lung consolidation in mechanically ventilated patients with and without ventilator-associated pneumonia: A prospective cohort study. J Crit Care 30: 327-333.

10. Wang G, Ji X, Xu Y, Xiang X (2016) Lung ultrasound: A promising tool to monitor ventilator-associated pneumonia in critically ill patients. Crit Care 20: 320

11. Reissig A, Copetti R (2014) Lung ultrasound in communityacquired pneumonia and in interstitial lung diseases. Respiration 87: 179-189.

12. Zagli G, Cozzolino M, Terreni A, Biagioli T, Caldini AL, et al. (2014) Diagnosis of ventilator-associated pneumonia: A pilot, exploratory analysis of a new score based on procalcitonin and chest echography. Chest 146: 1578-1585. 